\title{
Mezcla de Rocas para la Elaboración de un Sustrato Rico en Nutrientes
}

\author{
Mixture of rocks for the Preparation of a Nutrient-rich Substrate \\ Anakaren Flores-Téllez Girón (Da,*, Alejandra S. Gama-Castro ${ }^{(\mathbb{D} a}$, Felipe Legorreta-García ${ }^{\text {a a }}$, \\ Sandra L. Hernández-Flores iDa \\ a Área Académica de Ciencias de la Tierra y Materiales, Universidad Autónoma del Estado de Hidalgo, 42184, Pachuca, Hidalgo, México.
}

\begin{abstract}
Resumen
En este trabajo de investigación se realiza un estudio de dos tipos de rocas: zeolítica y fosfórica. Se realiza la caracterización de cada una mediante difracción de Rayos X, Microscopia Electrónica de Barrido, y Fluorescencia de Rayos X en dispersión de energía. Una de las rocas zeolíticas es intercambiada por iones amonio para lograr la obtención de iones disponibles dentro de la estructura de los cristales de zeolita. Los resultados indican que al realizar una mezcla de estas tres rocas se logra obtener un sustrato que puede ser capaz de sustituir los fertilizantes químicos para los diferentes tipos de plantas, basándonos en que la nutrición de estas dependen de tres elementos esenciales como el Nitrógeno, Fósforo y Potasio, estos proporcionan el material de construcción para los tejidos vegetales, las proporciones entre ellos van a variar según el tipo de cultivo; al conocer por medio de los estudios anteriormente mencionados los componentes de cada elemento en estas rocas podemos construir un sustrato según lo que se desea cultivar.
\end{abstract}

Palabras Clave:

Roca feldespática, Roca fosfórica, clinoptilolita, heulandita.

\begin{abstract}
In this research work, two types of rocks were studied: zeolitic and phosphoric rocks. The characterization of each one is carried out by X-ray diffraction, Scanning Electron Microscopy, chemical analysis by X-Ray Fluorescence. One of the zeolitic rocks is exchanged for ammonium ions to obtain available ions within the zeolite crystal structure. The results indicated that by mixing these three types of rocks, it is possible to obtain a substrate that can be able to substitute chemical fertilizers for different plants, based on the fact that plant nutrition depends on three essential elements such as Nitrogen, Phosphorus and Potassium. These provide the construction material for plant tissues, the proportions between them will vary according to the type of crop; by knowing through the above mentioned studies the components of each element in these rocks we can build a substrate according to what we want to cultivate.
\end{abstract}

Keywords:

Feldspathic rock, Phosphoric rock clinoptilolite, heulandite.

\section{Introducción}

México es un país mega diverso que constituye el $70 \%$ de la diversidad mundial de flora y fauna; un ejemplo son las suculentas, plantas más representativas de nuestro país que garantizan el cuidado y goce de un ambiente adecuado para el desarrollo y bienestar de los ecosistemas. En nuestro país se encuentra el mayor número de especies endémicas de estas plantas, por ejemplo, de 1400 especies de cactáceas que existen en el mundo, nuestro país alberga 669 lo que representa casi la mitad y 518 son endémicas de nuestro país; por otro lado el género Echeveria que pertenece a la familia Crassulaceae y que crece de manera silvestre en el norte del continente americano, consta de aproximadamente 154 especies, cerca de 132 existen en México es decir que el $85 \%$ de esta especie crece solo en nuestro país; la mayor riqueza y endemismo de especies se distribuye en el sureste del país, Oaxaca posee la mayor cantidad de especies (47), le siguen Puebla e Hidalgo con 19 y 18 especies respectivamente (SEMARNAT 2016). Sin embargo, actualmente en nuestro país enfrentamos una lamentable disminución de la biodiversidad y el

\footnotetext{
*Autor para la correspondencia: fl215489@uaeh.edu.mx García), hsandra.flores97@gmail.com (Sandra L. Hernández-Flores).

Correo electrónico: f1215489@uaeh.edu.mx (Anakaren Flores-Téllez Girón), ga354155@uaeh.edu.mx (Alejandra S. Gama-Castro), profe_974@uaeh.edu.mx (Felipe Legorreta- 
género de las suculentas está seriamente amenazado; sus poblaciones silvestres han sido afectadas por diversas actividades humanas como el uso del suelo en la agricultura, ganadería, así como la creciente urbanización, la sobreexplotación, su crecimiento lento en la mayoría de especies y la extracción de ejemplares para el comercio ilegal. Las suculentas tienen una gran demanda debido a su uso ornamental. Las plantas suculentas se distinguen por su capacidad de almacenar agua en los tallos, hojas y raíces, en forma de jugos mucilaginosos (Reyes, 2009), también presentan metabolismo fotosintético, este consiste en que los estomas se cierran durante el día y se abren en la noche, reduciendo la pérdida de agua (González, 2012). Estas características les permiten sobrevivir y reproducirse en ambientes áridos donde la disponibilidad de agua es baja y el periodo de sequía es prolongado (González, 2012; Arias-Toledo et al., 2011). Para su cultivo se debe tener cuidado con los periodos de riego, tener un suelo poroso y permeable, para que el exceso de agua se pierda con rapidez y el aire llegue fácilmente a las raíces. Se requiere que el suelo tenga un $\mathrm{pH}$ de 6 y que esté libre de sustancias orgánicas en descomposición, pues esto facilita la pudrición de la planta. También se necesitan elementos nutritivos como potasio, fósforo y nitrógeno; se suele utilizar un sustrato $70 \%$ mineral y $30 \%$ orgánico. En el presente trabajo de investigación se elabora un sustrato $100 \%$ mineral a partir de rocas fosfóricas y feldespáticas, se pone en evidencia que esta mezcla de rocas satisface la nutrición de la planta.

\section{Metodología}

Las rocas feldespáticas estudiadas se obtuvieron de depósitos ubicados en el municipio de Zimapán, Estado de Hidalgo y en el municipio de Huayacocotla, estado de Veracruz (las cuales se intercambiaron con iones amonio). Las rocas fosfóricas fueron otorgadas por la empresa Industrias Riviem, ubicada en el municipio de Pacula, Hidalgo.

Todas las rocas se trituraron y se clasificaron hasta obtener un tamaño de partícula de $1 \mathrm{~mm}$ y posteriormente, se secaron en un horno a $378 \mathrm{~K}$ durante 24 horas.

Para realizar el intercambio con iones amonio, de la muestra de Veracruz, se utilizó la técnica descrita por Leyva-Ramos (Leyva Ramos et al., 2005), la cual consiste en saturar $0.5 \mathrm{~g}$ de material con iones de $\mathrm{Na}^{+}$y posteriormente realizar la saturación con iones de $\mathrm{NH}^{4+}$.

La roca fosfórica es llevada a la quebradora de quijadas marca Allis Mineral, y posteriormente alimentado la quebradora de rodillos marca Quinn se obtiene un mineral con tamaño -3/8 para pasar por el pulverizador marca Holmes. Se toma una muestra representativa del material y se reduce de tamaño a -200 para los análisis de caracterización. Posteriormente, el resto de la muestra pasa al tamizador de laboratorio Ro-Tap, utilizando mallas de distintas aberturas serie ASTM (\#10, \#14, \#16, \#20, \#30, \#50, $\# 100$ y \#200). El material a utilizar con tamaño -\#30 +\#40 se tamizó en húmedo y posteriormente se secó a $100^{\circ} \mathrm{C}$ durante 12 horas, obteniendo así las rocas fosfóricas MI-1 y MI-2, posteriormente fueron analizadas por separado, pero se utilizó una alícuota de $50 \%$ de la roca MI-1 y 50\% en peso seco de la MI-2 para formar una muestra compuesta.

Finalmente, se realizó la mezcla de rocas feldespáticas y fosfóricas con las proporciones adecuadas, hasta obtener una mezcla homogénea por el método del cono que consiste en poner la muestra sobre una superficie plana, a la cual se le dará forma de pastel, y será dividida posteriormente en 4 partes lo más simétricas posible, luego se eligen dos lados opuestos con los que trabajará, y los dos restantes serán desechados (Jaime Simpson 2013).

\subsection{Caracterización}

Las muestras precursoras fueron analizadas por diferentes técnicas: La composición química se realizó mediante fluorescencia de rayos X (FRX) con un espectrómetro RIGAKU ZSX Primus II. Así mismo se analizaron mediante difracción de rayos X con un difractómetro marca INEL modelo Equinox 2000, los difractogramas se obtuvieron en un intervalo $2 \theta$ de $5^{\circ} \mathrm{a} 110^{\circ}$, la fuente de irradiación fue $\operatorname{Co}-\mathrm{K} \alpha 1 \quad(\lambda=1.789010 \AA)$, con un detector curvo, la distancia entre canales fue de $0.032 \theta$, aplicando $20 \mathrm{~mA}$ de corriente y un voltaje de $30 \mathrm{kV}$, el tiempo de irradiación fue de 30 minutos por muestra. La morfología de las partículas se estudió con un microscopio electrónico de barrido (MEB) marca JEOL modelo JSM-IT300, con una distancia de trabajo de $9.2 \mathrm{~mm}$ y un voltaje de aceleración de $20.0 \mathrm{kV}$, equipado con un detector de energía retrodispersiva de rayos X (EDS).

\section{Resultados y Discusión}

\subsection{Análisis de las rocas fosfóricas por Espectrometría de Fluorescencia de rayos $X$ (FRX)}

Se determinaron por medio de esta técnica de caracterización los elementos mayoritarios y a partir se éstos se determinó los óxidos en ambas muestras de la roca fosfórica (Tabla 1); posteriormente se tomó en cuenta el promedio entre MI-1 y MI-2. El análisis de FRX fue realizado una sola vez para cada muestra.

Tabla 1: Elementos y óxidos mayoritarios de las rocas fosfóricas

\begin{tabular}{cccc}
\hline Elemento & MI-1 & MI-2 & $\begin{array}{c}\text { \% en } \\
\text { abundancia }\end{array}$ \\
\hline $\mathrm{SiO}_{2}$ & 12 & 5.23 & 8.615 \\
$\mathrm{TiO}_{2}$ & 0.01 & 0.058 & 0.034 \\
$\mathrm{Al}_{2} \mathrm{O}_{3}$ & 1.31 & 1.91 & 1.61 \\
$\mathrm{Fe}_{2} \mathrm{O}_{3}$ & 0.683 & 0.568 & 0.6255 \\
$\mathrm{MnO}$ & 0.16 & 0.064 & 0.112 \\
$\mathrm{MgO}$ & 0.061 & 0.068 & 0.0645 \\
$\mathrm{CaO}$ & 46.9 & 51.6 & 49.25 \\
$\mathrm{Na} 2$ & 0.11 & 0.095 & 0.1025 \\
$\mathrm{~K}_{2} \mathrm{O}$ & 0.079 & 0.051 & 0.065 \\
$\mathrm{P}_{2} \mathrm{O}_{5}$ & 33.7 & 32.5 & 33.1 \\
$\mathrm{~F}$ & 2.91 & 2.46 & 2.685 \\
$\mathrm{SO}_{3}$ & 0.288 & 0.282 & 0.285 \\
\hline
\end{tabular}

\subsection{Microscopía electrónica de barrido y espectroscopia de energía dispersiva ( $M E B \& E D S)$.}

La micrografía de la muestra MI-1 (Figura 1a) exhibe una morfología idiomorfa, los cristales presentan una forma prismática hexagonal con tamaños entre $2 \mu \mathrm{m}$ en la base y $8 \mu \mathrm{m}$ de altura; la base hexagonal que presentan es común de una apatita. Al no encontrar contenidos de $\mathrm{F}$ en el análisis EDS (Figura $1 \mathrm{~b})$, es posible que pueda tratarse de una hidroxiapatita; existe una sustitución de iones de $\left(\mathrm{OH}^{-}\right),\left(\mathrm{Ca}^{2+}\right)$ y $\left(\mathrm{PO}_{4}{ }^{-3}\right)$. En la (Figura 1c) se puede observar una matriz de cristales idiomorfos que presentan una forma prismática rectangular con un tamaño menor a $10 \mu \mathrm{m}$, y sobre ellos también se observan partículas en forma de hojuelas con tamaños menores a $10 \mu \mathrm{m}$. 


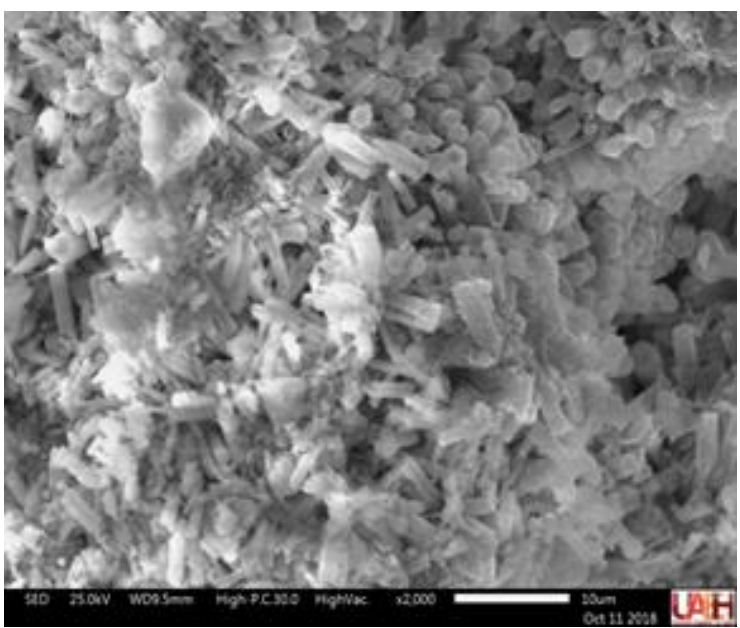

Figura. 1a) Micrografía de MI-1 a 2000X.

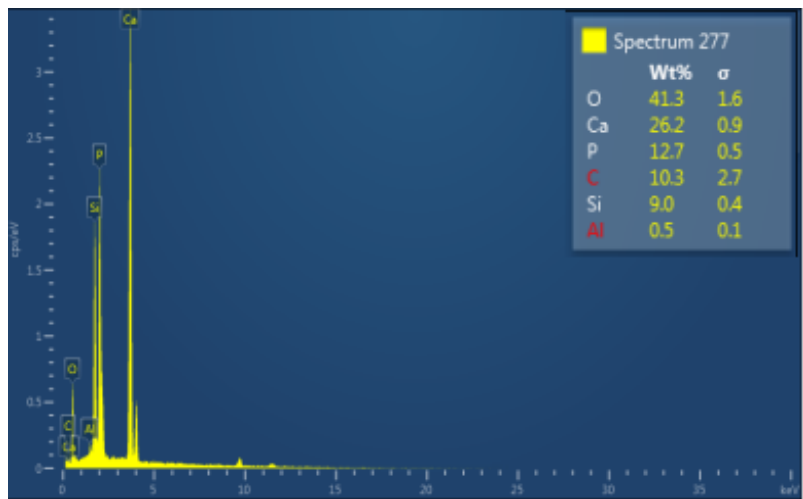

Figura 1b) Análisis EDS para la muestra MI-1

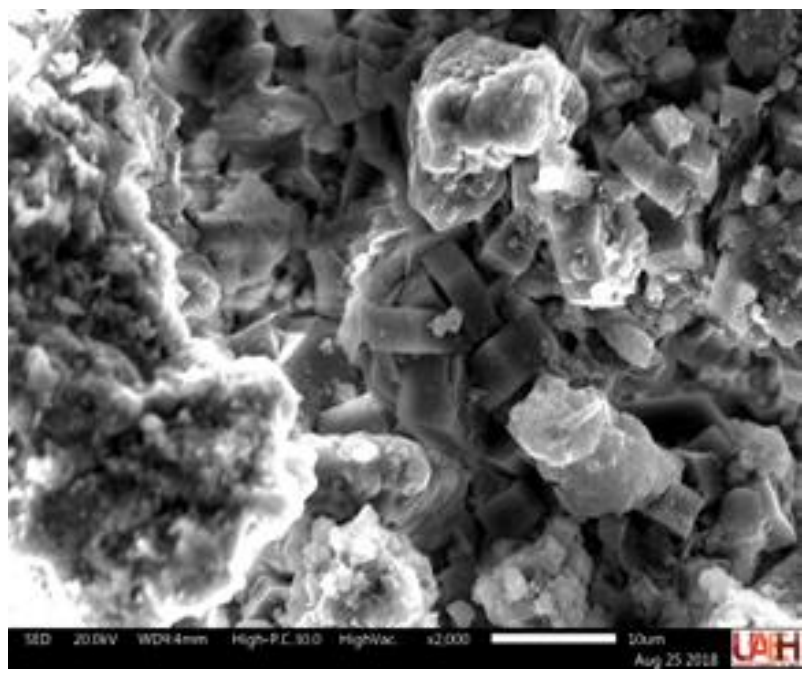

Figura 1c) Micrografía de MI-1 a 2000X

En la (Figura 2a) se presenta la micrografía de la muestra MI2 que revela la presencia de partículas subredondeadas con un tamaño menor a $10 \mu \mathrm{m}$, en la (Figura 2b) se observan cristales más definidos y con una forma de prismas hexagonales con tamaños entre 1 y $2 \mu \mathrm{m}$. Estos presentan la siguiente composición química: $\mathrm{O}=53.1 \%, \mathrm{Ca}=20.4 \%, \mathrm{P}=10.8 \%, \mathrm{~F}=8.6 \%, \mathrm{C}=6.9 \%$ y $\mathrm{Si}=0.2 \%$.

De acuerdo al análisis con EDS (Figura 2c), se exhibe cierto grado de sustitución en mayor o menor grado. La morfología de las muestras MI-1 y MI-2 son partículas subredondeadas con un tamaño menor a $10 \mu \mathrm{m}$ y sobre éstas se presentan pequeñas partículas con forma de hojuela. También se revelaron cristales con morfología idiomorfa y prismática en ambas muestras.

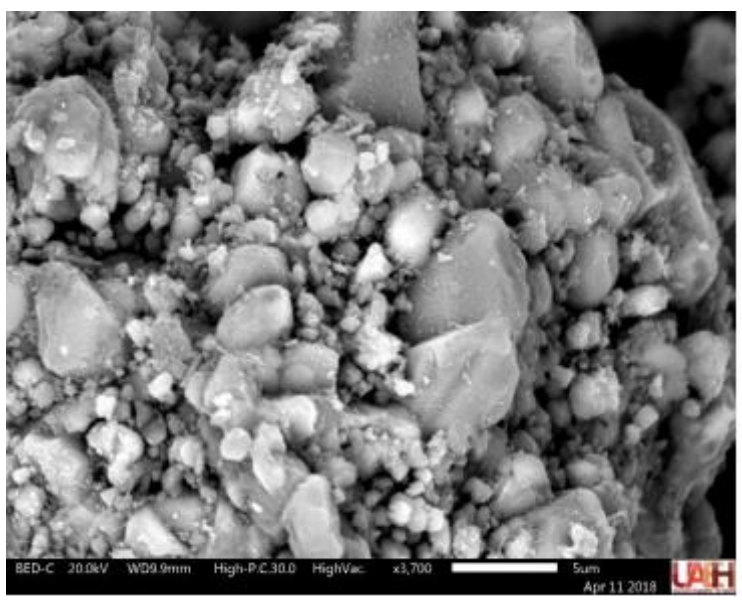

Figura 2a) Micrografía de MI-2 a 3700X

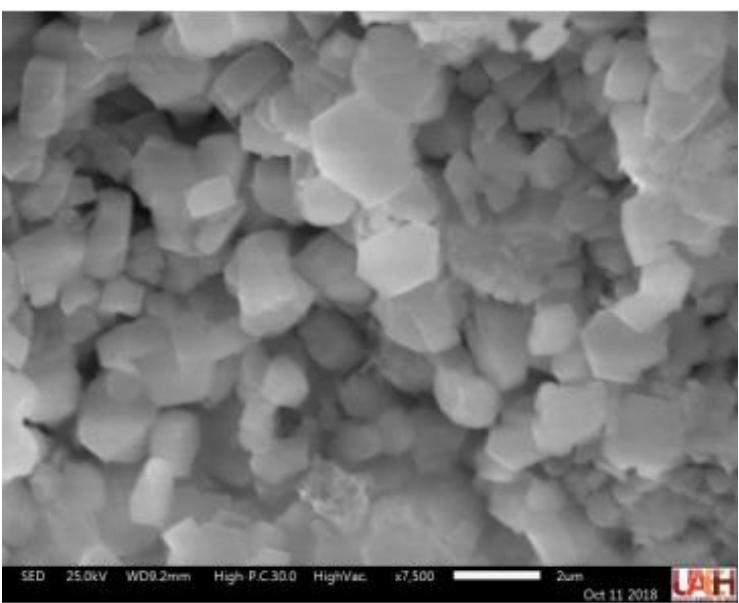

Figura 2b) Micrografía de MI-2 a 7500X

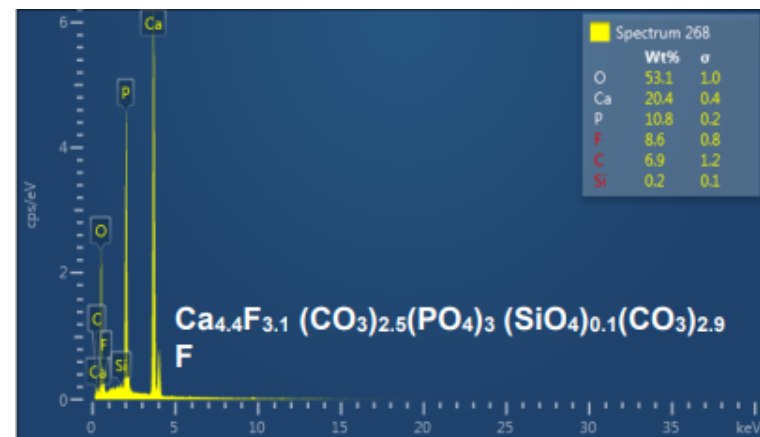

Figura 2c) Análisis EDS para la muestra MI-2

\subsection{Difracción de Rayos $X(D R X)$}

Por medio de este análisis, se pudieron detectar las especies mineralógicas correspondientes a la fluorapatita $\mathrm{Ca}_{9.3} \mathrm{Mn}_{0.7}\left(\mathrm{PO}_{4}\right)_{6}$ $2 \mathrm{~F}$ y $\mathrm{Ca}_{10}\left(\mathrm{PO}_{4}\right)_{6} \mathrm{~F}$, cuarzo $\mathrm{SiO}_{2}$ y calcita $\left(\mathrm{CaCO}_{3}\right)$, como se muestra en el difractograma de la figura 3 y en Tabla 2.

Para la muestra MI-1 (Figura 3) las intensidades de la apatita se ven afectadas por los comportamientos de las reflexiones, que podría deberse al acomodo de los átomos en la estructura cristalina 
o a la pérdida de $\mathrm{CO}_{2}$. Se detecta un pico fosfatado sin su posible identificación; esta muestra cuenta con $1.31 \%$ de $\mathrm{Al}_{2} \mathrm{O}_{3}$ y $0.683 \%$ de $\mathrm{Fe}_{2} \mathrm{O}_{3}$ y el contenido de flúor es mayor.

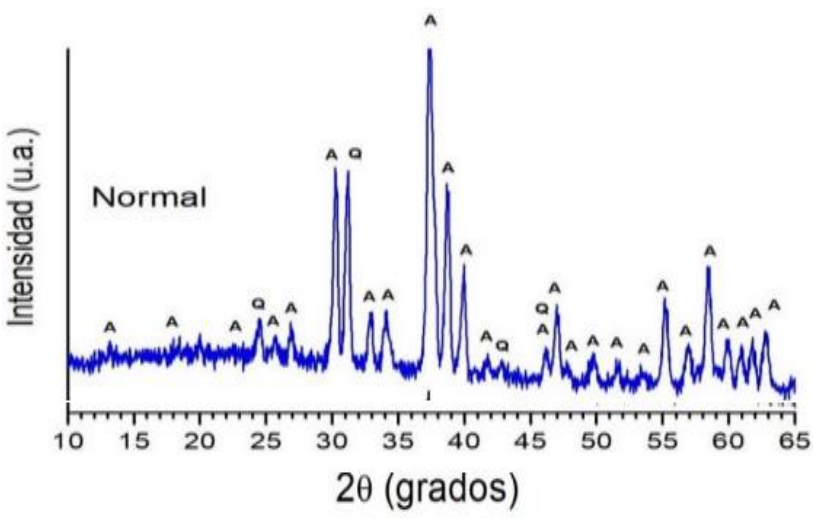

Figura 3. Difracción de rayos $\mathrm{X}$ de la muestra MI-1

Tabla 2: Especies mineralógicas identificadas a partir de DRX

\begin{tabular}{ccclcc}
\hline Mineral & $\begin{array}{c}\text { PDF-02 } \\
\text { (ICDD) }\end{array}$ & Símbolo & $\begin{array}{l}\text { Fórmula } \\
\text { Química }\end{array}$ & $\begin{array}{c}\text { Muestra } \\
\text { MI-1 MI-2 }\end{array}$ \\
\hline Fluorapatita & $77-1902$ & $\mathrm{~A}$ & $\begin{array}{l}\mathrm{Ca}_{9.3} \mathrm{Mn}_{0.7} \\
\left(\mathrm{PO}_{4}\right)_{6} 2 \mathrm{~F}\end{array}$ & $\mathrm{x}$ & \\
Fluorapatita & $71-0881$ & $\mathrm{~A}$ & $\mathrm{Ca}_{10}\left(\mathrm{PO}_{4}\right)_{6} \mathrm{~F}$ & & $\mathrm{x}$ \\
Cuarzo & $83-2468$ & $\mathrm{Q}$ & $\mathrm{SiO}_{2}$ & $\mathrm{x}$ & \\
Cuarzo & $85-0865$ & $\mathrm{Q}$ & $\mathrm{SiO}_{2}$ & & $\mathrm{x}$ \\
Calcita & $72-1937$ & $\mathrm{C}$ & $\mathrm{Ca} \mathrm{CO}_{3}$ & & x \\
\hline
\end{tabular}

La muestra MI-2 (Figura 4) contiene calcita y, los nuevos picos formados son de óxido de calcio $(\mathrm{CaO})$; esta muestra cuenta con $1.91 \%$ de $\mathrm{Al}_{2} \mathrm{O}_{3}$ y $0.568 \%$ de $\mathrm{Fe}_{2} \mathrm{O}_{3}$.

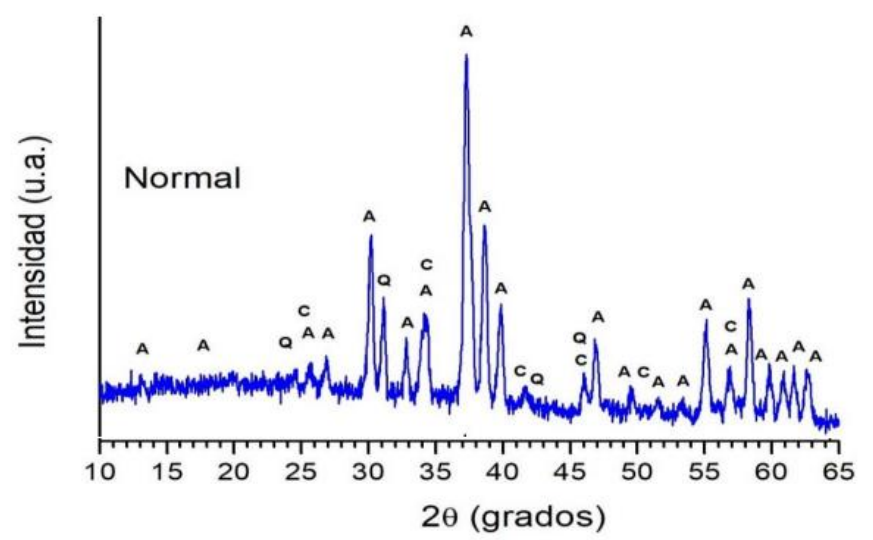

Figura 4 Difracción de rayos $\mathrm{X}$ de la muestra MI-2

\subsection{Análisis de las rocas Feldespáticas por Espectroscopia de Fluorescencia de rayos $X(X R F)$.}

Mediante este análisis se obtuvieron los elementos mayoritarios de la muestra $\mathrm{H}-1$ perteneciente al municipio de Zimapán, estado de Hidalgo y para la muestra V-3 perteneciente al municipio de Huayacocotla, estado de Veracruz, presentados a continuación (Tabla. 3). Como se puede observar en la tabla 3, en las muestras $\mathrm{H}-1$ y V-3, el mayor contenido es de $\mathrm{SiO}_{2}$ es $67.424 \%$ y $70.021 \%$ respectivamente, seguido del $\mathrm{Al}_{2} \mathrm{O}_{3}$ con $12.483 \%$ y $11.047 \%$ respectivamente.
Tabla 3: Elementos y óxidos mayoritarios de las Rocas Feldespáticas.

\begin{tabular}{cccc}
\hline Elementos & $\mathrm{H}-1$ & $\mathrm{~V}-3$ & $\begin{array}{c}\text { \% en } \\
\text { abundancia }\end{array}$ \\
\hline $\mathrm{SiO}_{2}$ & 67.424 & 70.021 & 5.23 \\
$\mathrm{TiO}_{2}$ & 0.257 & 0.105 & 0.058 \\
$\mathrm{Al}_{2} \mathrm{O}_{3}$ & 12.483 & 11.047 & 1.91 \\
$\mathrm{Fe}_{2} \mathrm{O}_{3} \mathrm{t}$ & 2.446 & 1.479 & 0.568 \\
$\mathrm{MnO}$ & 0.039 & 0.019 & 0.064 \\
$\mathrm{MgO}$ & 1.562 & 1.448 & 0.068 \\
$\mathrm{CaO}$ & 2.566 & 1.784 & 51.6 \\
$\mathrm{Na}_{2} \mathrm{O}$ & 0.891 & 0.435 & 0.095 \\
$\mathrm{~K}_{2} \mathrm{O}$ & 3.15 & 3.253 & 0.051 \\
$\mathrm{P}_{2} \mathrm{O}_{5}$ & 0.072 & 0.026 & 32.5 \\
\hline
\end{tabular}

3.5. Microscopía electrónica de barrido y espectroscopia de energía dispersiva ( $M E B \& E D S)$.

Las micrografías de los polvos de las rocas feldespáticas se presentan en las Figuras 5 y Figuras 6a-c. En la Figura 5 se observa que éstas consisten en partículas de diferente morfología haciendo alusión a la presencia de distintas arcillas y posibles cristales de clinoptilolita con tamaños inferiores a los $10 \mu \mathrm{m}$.

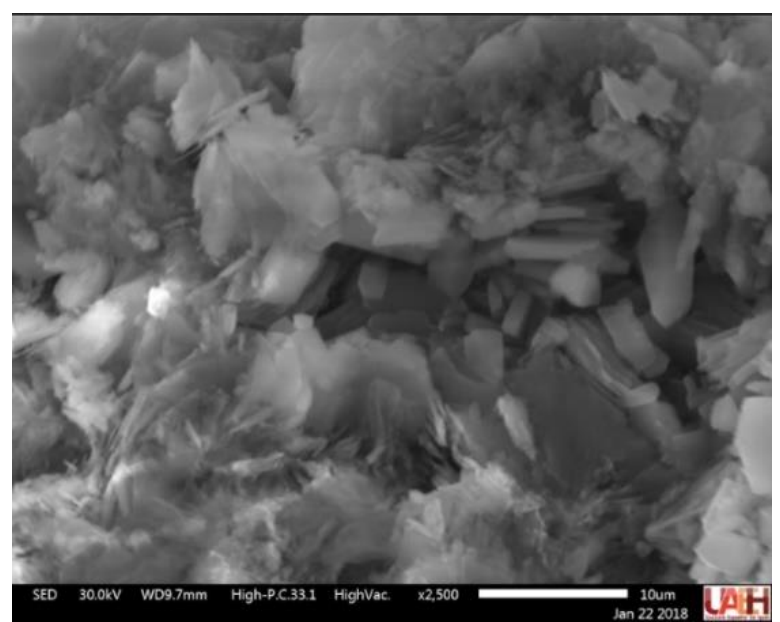

Figura 5. Micrografías de las rocas feldespáticas

En la Figura 6a se observa una partícula de forma subhedral y de hábito prismático, que mide alrededor de $30 \mu \mathrm{m}$ de diámetro. El análisis químico en una zona concreta de esta partícula, contiene $\mathrm{SiO}_{2} 80,29 \%, \mathrm{Al}_{2} \mathrm{O}_{3}: 8,98 \%, \mathrm{Fe}_{2} \mathrm{O}_{3}: 4,15 \%$ y $\mathrm{CaO}: 3,26 \%$ wt.

\subsection{Análisis químico de partículas}

Los bajos contenidos de Al pueden indicar que el ion Fe está ubicado en sitios octaédricos de cristales de zeolita. En la Figura $6 \mathrm{~b}$ se observa una partícula de esméctita con un tamaño aproximado de $40 \mu \mathrm{m}$, de forma euhédrica y un hábito de copos, típico de las arcillas. En el centro de esta partícula se observan cristales de zeolita típicos de 5-10 $\mu \mathrm{m}$ de longitud, de forma euhédrica y hábito acicular. El análisis químico de EDS aplicado en la esmectita, indica que es un tipo de montmorillonita $\mathrm{Ca}-\mathrm{Na}$. En la Figura 6c se muestra un tectosilicato con una longitud de aproximadamente $100 \mu \mathrm{m}$. En la porción central se aprecia una partícula con una fractura concoidal, característica de los materiales amorfos. 
Considerando que el análisis EDS aplicado a esta partícula fue de $97.99 \%$ en peso de $\mathrm{SiO}_{2}$ y su amorfismo, la muestra puede atribuirse como ópalo, probablemente producto de una alteración epitermal.

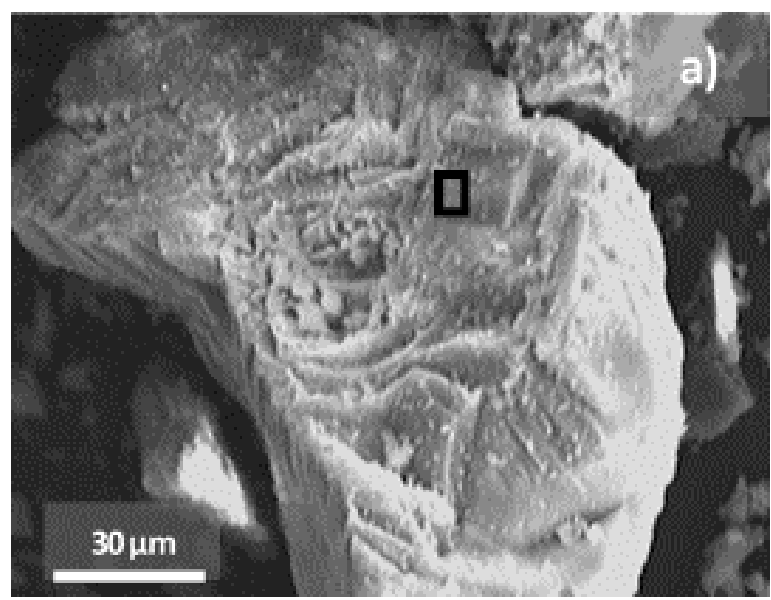

Figura 6a. Micrografías de los polvos de las rocas feldespáticas.

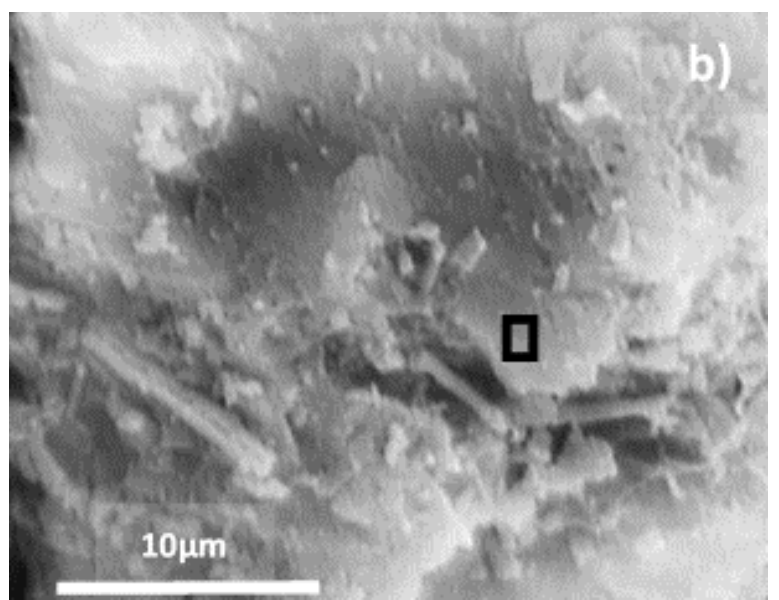

Figura 6b. Micrografías de los polvos de las rocas feldespáticas.

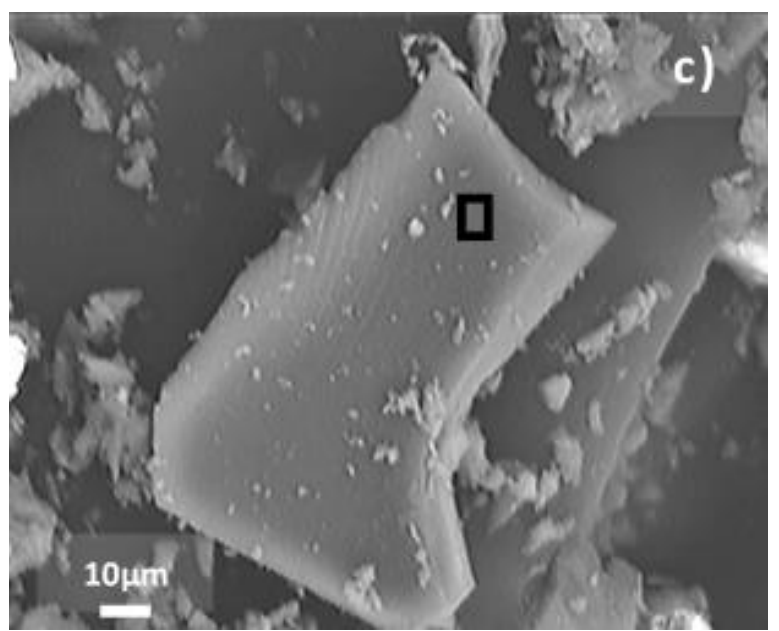

Figura 6c. Micrografías de los polvos de las rocas feldespáticas.
Tabla 4: Análisis de los componentes químicos

\begin{tabular}{cc|c|c|c|c|c|c|c}
\hline $\begin{array}{c}\text { Superficie del } \\
\text { cuadrado }\end{array}$ & \multicolumn{7}{c}{ \% en peso de los componentes } \\
\cline { 2 - 10 } & $\mathrm{SiO}_{2}$ & $\mathrm{Al}_{2} \mathrm{O}_{3}$ & $\mathrm{~K}_{2} \mathrm{O}$ & $\mathrm{CaO}$ & $\mathrm{Fe}_{2} \mathrm{O}_{3}$ & $\mathrm{MgO}$ & $\mathrm{Na}_{2} \mathrm{O}$ & $\mathrm{TiO}$ \\
\hline a) & 80.29 & 8.98 & 1.89 & 3.26 & 4.15 & 0.86 & 0.57 & -- \\
b) & 56.51 & 13.63 & 2.32 & 10.6 & 5.1 & 7.04 & 4.71 & 0.1 \\
c) & 97.99 & 1.28 & 0.12 & 0.19 & 0.16 & -- & 0.26 & -- \\
\hline
\end{tabular}

\subsection{Difracción de Rayos $X(D R X)$}

Los patrones de DRX de los polvos preparados se muestran en la figura 7; es posible distinguir dos fases cristalinas: $\mathrm{Z}=$ zeolita (heulandita-Ca) y $\mathrm{C}=$ baja cristobalita. También se encontraron otros tipos de arcillas y feldespatos como Sanidina-Fe, montmorillonita-18- $\AA$, chamosita, illita y andesina.

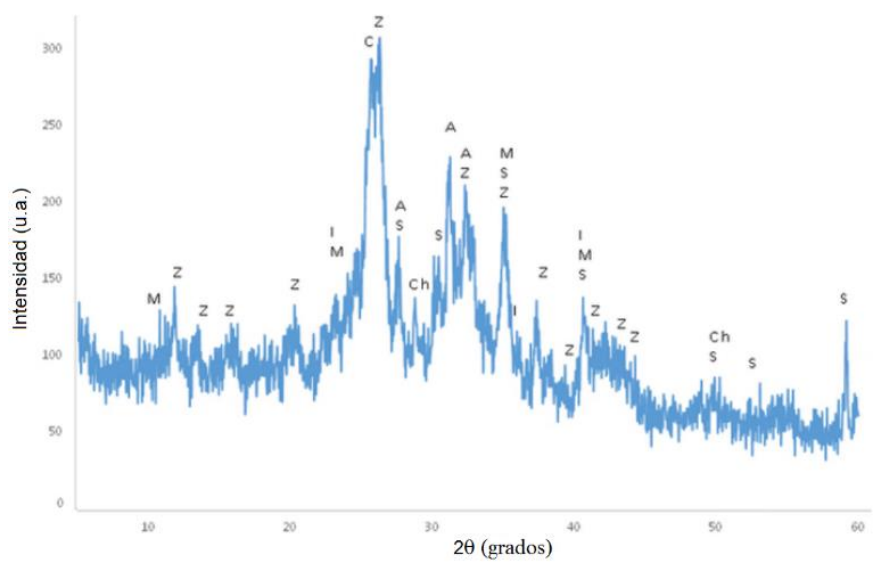

Figura 7. Patrones de difracción de rayos $\mathrm{X}$ de las rocas feldespáticas.

\section{Conclusiones}

Se logró obtener una mezcla de roca fosfórica mezclada en una proporción de $80 \%$ en peso de zeolita natural, $15 \%$ de zeolita intercambiada con $\mathrm{NH}_{4}$ y $5 \%$ de roca fosfórica, esta mezcla fue elaborada con las proporciones de elementos que se encuentran en los fertilizantes que proporcionan el material de construcción para los tejidos vegetales $\mathrm{N}, \mathrm{P}$ y K en una proporción de 122 (una parte de $\mathrm{N}$, por dos partes de $\mathrm{P}$ y dos de $\mathrm{K}$ ). Las rocas fueron caracterizadas por medio de las técnicas de fluorescencia de rayos $\mathrm{X}$, difracción de rayos $\mathrm{X}$ y análisis químico, comprobando la presencia de fosforita y clinoptilolita. Este sustrato evitaría la pudrición de la planta proporcionando los nutrientes requeridos.

\section{Referencias}

Arias-Toledo, A.; M.T. Valverde-Valdés, y J. Reyes-Santiago. (2011). Las plantas de la región de Zapotitlán Salinas, Puebla. Secretaría de Medio Ambiente, Recursos Naturales y Pesca, Instituto Nacional de Ecología. México. 80 pp.

Cabrera, N. R. (2007). Fundamentos de química analítica básica. Colombia: Universidad de Caldas, Ciencias Exactas y Naturales.

C. Baerlocher, L. B. McCusker, y D. H. Olson, Atlas of zeolite framework types. Elsevier, 2007.

D. L. Bish y J. M. Boak, "Clinoptilolite-Heulandite Nomenclature”, Rev. Mineral. Geochem., vol. 45, núm. 1, pp. 207-216, ene. 2001.

F. A. MuuproN, "CLINOPTILOLITE REDEFINED”, p. 19. 
F. PECHAR y D. RYKL, "Study of the thermal stability of the natural zeolite heulandite", pp. 9 ..

González, M.F., 2012. Las zonas áridas y semiáridas de México y su vegetación. México DF, 194.

J. F. Marco, M. Gracia, J. R. Gancedo, T. González-Carreño, Arcoya, A., \& Seoane, $\quad$ X. L. "On the state of iron in a clinoptilolite". Hyperfine Interactions, vol. 95(1), pp. 53-70, 1995.

Leyva-Ramos, R.; Medellín-Castillo, N. A.; Guerrero-Coronado, R. M. Berber-Mendoza, M. S.; Aragón-Piña, A.; Jacobo-Azura, A. Intercambio Iónico de Plata (I) en solución acuosa sobre Clinoptilolita. 2005, 4, 193200.
Reyes, S.J., 2009. Manual práctico de Conservación y Restauración de Cactáceas y otras Plantas Suculentas. Comisión Nacional Forestal. Secretaría de Medio Ambiente y Recursos Naturales. México, 108 pp.

Secretaría de Medio Ambiente y Recursos Naturales (2016), Cactáceas, riqueza natural de México.

Simpson Jaime, Técnicas de muestreo, Chile, 2013.

S. M. Auerbach, K. A. Carrado, y P. K. Dutta, Handbook of zeolite science and technology. CRC press, 2003. 